\title{
Spatial learning as activator of hippocampal neurogenesis during aging and development of Alzheimer's disease-like pathology
}

\author{
Alena Burnyasheva \\ Molecular mechanisms of aging \\ Institute of Cytology and Genetics \\ SB RAS, Novosibirsk, Russia \\ burnyasheva@bionet.nsc.ru
}

\author{
Tatiana Kozlova \\ Molecular mechanisms \\ of aging \\ Institute of Cytology and Genetics \\ SB RAS, Novosibirsk, Russia \\ kozlova@bionet.nsc.ru \\ Natalia Stefanova \\ Molecular mechanisms of aging Institute \\ of Cytology and Genetics SB RAS, \\ Novosibirsk, Russia \\ stefanovan@bionet.nsc.ru
}

\author{
Ekaterina Rudnitskaya \\ Molecular mechanisms of aging \\ Institute of Cytology and Genetics \\ SB RAS, Novosibirsk, Russia \\ rudnickaya@bionet.nsc.ru
}

\begin{abstract}
Adult neurogenesis in dentate gyrus (DG) is one of the key mechanisms of neuronal plasticity in hippocampus and plays an important role in cognitive function. However, the consequences of its alteration during healthy aging as well as development of neurodegeneration including Alzheimer's disease (AD) remain unclear. It was shown that factors which can activate neurogenesis - such as physical exercises and learning - are able to improve cognitive function. Animal models are useful to clarify the connection between adult neurogenesis and cognitive function during development of AD signs, and OXYS rats are a suitable model for the most common sporadic form of AD. Here we examined effects of spatial learning on neurogenesis in DG of OXYS rats prior to and during manifestation of AD signs. We showed altered reference memory of OXYS rats already at the period prior to neurodegeneration. At the period of active manifestation of AD signs OXYS rats demonstrated altered spatial learning and reversal learning, whereas reference memory was altered only a little. At the period of active amyloid- $\beta$ accumulation in the brain only reference memory of OXYS rats was altered. Spatial learning resulted in accelerated maturation of immature cells of neuronal and astrocytic cell lineages in DG of OXYS and Wistar rats and decrease of amyloid- $\beta$ content in aged animals.
\end{abstract}

Keywords - neurogenesis, spatial learning, Alzheimer's disease, OXYS rats

\section{Introduction}

Alzheimer's disease (AD) is detrimental multifactorial disorder developing asymptomatically for many years prior to its manifestation [1]. There is no effective cure for $\mathrm{AD}$ to date, however interventions prior to manifestation of the disease symptoms are able to improve cognitive function in patients with mild cognitive impairment and thus to slow down or even prevent development of AD [2]. Indeed, several studies revealed positive associations of physical activity, cognitive training or both with cognition in elderly people and patients with mild cognitive impairment [3-5]. These cognitive improvements may be achieved because of activation of neuroplasticity: it is well known, that physical exercises and cognitive training result in activation of neurogenesis in hippocampal DG of adult animals [6]. However, the precise mechanisms underlying cognitive improvement in aged animals and, moreover, elderly people are still unknown, and its examination requires the use of suitable animal models for human neurodegenerative disorders.
Previously we have shown that senescence-accelerated OXYS rats may be considered as an adequate model of the late-onset sporadic AD because of disease signs are developed spontaneously without mutations in App, Psen 1 and Psen 2 genes. First neurodegenerative changes occur in OXYS rats at 3 months of age, and active amyloid- $\beta$ accumulation in the brain observed at 12 months of age [7].

\section{Materials and methods}

To evaluate spatial learning, reversal learning and reference memory of OXYS and control Wistar rats at 1.5, 3 and 12 months of age ( $\mathrm{n}=8$ animals per strain and age) we used Morris water maze. To analyze changes in hippocampal neurogenesis of OXYS and Wistar rats we evaluated density of progenitors and cells from neuronal and astrocytic lineages $(n=3$ to 6 per group, strain and age) in DG and the content of amyloid- $\beta$ by immunohistochemistry using antibodies specific for molecular markers of various cell types as well as for amyloid- $\beta$.

\section{Results}

Decrease of reference memory in OXYS rats was observed already at 1.5 months of age; however, spatial learning and reversal learning did not differ compared to Wistar rats. At the period of active manifestation of AD meaning 3 months of age OXYS rats demonstrated totally altered spatial learning and reversal learning, whereas reference memory was altered only a little. We observed decrease of reference memory in OXYS rats at stage of amyloid- $\beta$ accumulation in the brain (12 months of age); however, we did not show any differences in learning and reversal learning abilities between OXYS and Wistar rats at this age. Learning in the Morris water maze from 1.5 months of age resulted in accelerated maturation of immature cells of neuronal and astrocytic cell lineages in Wistar rats and only immature cells of neuronal cell lineage in OXYS rats. Learning from 3 months of age accelerated maturation of immature cells of neuronal lineage in Wistar rats as well as activated astrocytogenesis in both OXYS and Wistar rats. Learning from 12 months of age did not affect cell densities in DG of Wistar rats and resulted in accelerated maturation of immature cells of astrocytic lineage in hippocampal neurogenic niche in OXYS rats. Learning from 12 months of age also affected amyloid- $\beta$ content in DG: indeed, the parameter was lower in animals trained in Morris water maze. 


\section{Conclusion}

We demonstrated that spatial learning affected neurogenesis, however the impact depended on the age at which the animals were trained and the stage of AD-like pathology. Naturally, this positive effect was stronger for young adult rats and less clear when signs of AD in OXYS rats were already developed. Learning also lowered the content of amyloid- $\beta$ in the DG of aged animals. Thus, hippocampal-dependent learning may be considered as a perspective strategy to intensify neuronal plasticity and thereby improve cognitive function even in aged animals and probably in elder people.

\section{ACKNOWLEDGMENT}

Senescence-accelerated OXYS rats and Wistar rats were obtained from the Breeding Experimental Animal Laboratory of the Institute of Cytology and Genetics, SB RAS, Novosibirsk, Russia. The microscopy was conducted at the Multi-Access Center for Microscopy of Biological Objects (Institute of Cytology and Genetics, SB RAS, Novosibirsk, Russia).

\section{REFERENCES}

[1] M. Crous-Bou, C. Minguillón, N. Gramunt, J. L. Molinuevo, "Alzheimer's disease prevention: from risk factors to early intervention," Alzheimers Research Therapy, vol. 9, 2017, No. 71.
[2] G. K. Bhatti, A. P. Reddy, P. H. Reddy, J. S. Bhatti, "Lifestyle Modifications and Nutritional Interventions in Aging-Associated Cognitive Decline and Alzheimer's Disease," Frontiers in Aging Neuroscience, vol. 11, 2020, pp. 1-15.

[3] M. Roig, S. Nordbrandt, S. S. Geertsen, J. B. Nielsen, "The effects of cardiovascular exercise on human memory: A review with metaanalysis," Elsevier, vol. 37, 2013, pp. 1645-1666.

[4] A. Lampit, H. Hallock, M. Valenzuela, "Computerized cognitive training in cognitively healthy older adults: a systematic review and meta-analysis of effect modifiers," PLoS Med, vol.11, 2014, pp. 1-18.

[5] T. Ngandu, J. Lehtisalo, A. Solomon, E. Levalahti, S. Ahtiluoto, R. Antikainen et al., "A 2 year multidomain intervention of diet, exercise, cognitive training and vascular risk monitoring versus control to prevent cognitive decline in at-risk elderly people (FINGER): a randomised controlled trial," Lancet, vol. 385, 2015, pp. 2255-2263.

[6] P. Baptista and J. P. Andrade, "Adult Hippocampal Neurogenesis: Regulation and Possible Functional and Clinical Correlates,"Fronties in Neuroanatomy, vol.12, 2018, pp. 1- 23.

[7] N. A. Stefanova, O. S. Kozhevnikova, A. O. Vitovtov, K. Y. Maksimova, S. V. Logvinov, E. A. Rudnitskaya, E. E. Korbolina, N. A. Muraleva, N. G. Kolosova, "Senescence-accelerated OXYS rats: A model of agerelated cognitive decline with relevance to abnormalities in Alzheimer disease," Cell Cycle, vol. 13, 2014, pp. 898-909. 